\title{
MSU crystals and osteoblasts: insight into bone erosion in gout
}

"MSU crystals have impressive negative effects on the survival, function and differentiation of osteoblasts," says Nicola Dalbeth, from the University of Auckland, New Zealand. These claims are based on the findings of the latest paper from Dalbeth's group, published in Annals of the Rheumatic Diseases.

Bone erosion is common in patients with chronic tophaceous gout, but its cause is not well understood. Monosodium urate crystals (MSU) are thought to be involved, as collections of MSU crystals (tophi) occur in subchondral bone of patients with gout. Previously, the group focused predominantly on the role of osteoclasts in this setting. "We have shown that patients with erosive gout have disordered formation of osteoclasts, and that MSU crystals indirectly promote osteoclast formation through interactions with stromal cells such as osteoblasts," states Dalbeth. Here, the researchers focus on the role of osteoblasts in bone erosion.

First, the effects of MSU crystals on cultures of primary osteoblasts, and osteoblast-like cell lines were investigated.
MSU crystals had a dose-dependent influence on the survival of all cell types, resulting in reduced viability and cell death. As the effect was similar in primary osteoblasts and osteoblast-like cell lines, cell lines were used to investigate the effects of MSU crystals in more detail. Neither crystal length, nor the addition of serum to the culture medium, nor inhibition of crystal phagocytosis blocked the negative effect of the MSU crystals on cultured cells; in addition, although numbers of viable cells decreased, the numbers of cells undergoing apoptosis did not change on culture with MSU crystals, leading the authors to conclude that the cells were dying by another method, most probably necrosis.

Next, the authors performed long-term cultures of the osteoblast-like cells in mineralization assays and showed that MSU crystals inhibited mineralization and reduced the area of bone formed by these cells, in comparison with cells cultured in the absence of MSU crystals. Furthermore, the expression of genes related to osteoblast differentiation decreased on exposure to high levels of MSU crystals, in comparison

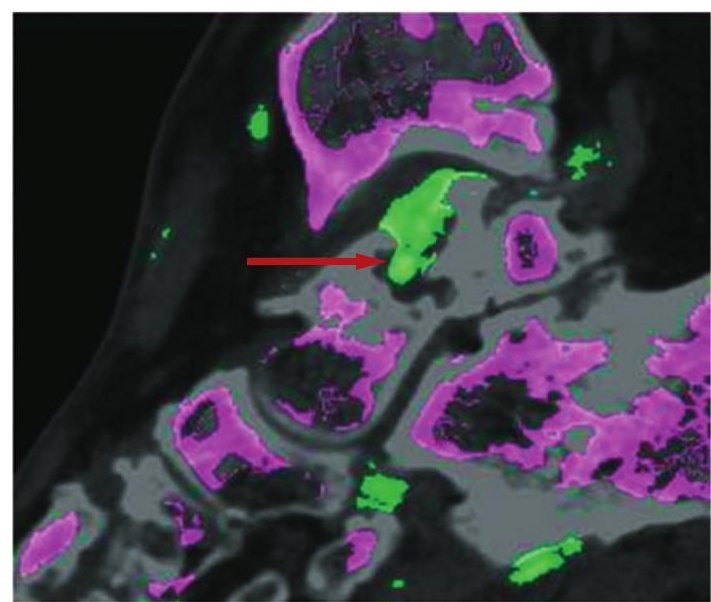

Dual energy CT scan of a patient with chronic tophaceous gout showing MSU crystals (green) in a bone erosion. Image courtesy of N. Dalbeth.

numbers of osteoblasts, compared with bone samples from areas not affected by MSU crystals. The authors conclude that reduced osteoblast differentiation and increased osteoclast formation contribute to bone erosion at the tophi-bone interface in gout.

Jenny Buckland

Original article Chhana, A. et al. Monosodium urate monohydrate crystals inhibit osteoblast viability and function: implications for development of bone erosion in gout. Ann. Rheum. Dis. doi:10.1136/ard.144774 